\title{
Associations with anticipated and obtained outcomes in instrumental learning
}

\author{
ROBERT A. RESCORLA and RUTH M. COLWILL \\ University of Pennsylvania, Philadelphia, Pennsylvania
}

\begin{abstract}
In four experiments, we investigated encoding of the reinforcer in instrumental learning. We contrasted the view that the reinforcer is encoded as a consequence of the response with the position that the expectation of the reinforcer serves as an antecedent stimulus for the response. In all four experiments, a response was followed by one reinforcer in the presence of a stimulus known to elicit an expectation of a different reinforcer. In Experiments 1 and 2, we found that devaluing the consequent reinforcer reduced performance of the response more than did devaluing the expected reinforcer. In Experiment 3, we found no evidence at all for a detrimental effect of devaluing the expected reinforcer. Experiment 4 showed that a stimulus associated with a reinforcer will preferentially promote a response that has the same-consequent reinforcer rather than the same-antecedent reinforcer. These results provide further support for the view that response-reinforcer associations are the crucial product in instrumental learning situations.
\end{abstract}

Classical S-R theories of instrumental learning explain performance primarily in terms of the development of an association between an antecedent stimulus $(S)$ and the instrumental response (R). According to these theories, the role of the consequent reinforcer is simply to promote the formation of that $\mathrm{S}-\mathrm{R}$ association. They make little provision for learning about the reinforcing outcome $(\mathrm{O})$ per se; $O$ only serves to foster the encoding of the relation between $S$ and $R$.

However, there are now available several kinds of evidence suggesting that learning about the reinforcer does occur in many instrumental learning situations (see Colwill \& Rescorla, 1986, for a recent review). For instance, in an elegant series of experiments, Trapold, Overmier, and their colleagues (e.g., Brodigan \& Peterson, 1976; Overmier, Bull, \& Trapold, 1971; Peterson, Wheeler, \& Trapold, 1980; Trapold, 1970) have found instrumental discrimination learning to be enhanced when the outcomes for different $S / R$ pairs are made discriminably different. This sensitivity to the differential reinforcer outcomes used during training implies some learning about the reinforcer itself. Relatedly, Colwill and Rescorla (1988) have recently reported that a stimulus whose response has been trained with a particular reinforcer more successfully transfers its control to other responses trained with that same outcome than to other responses trained with different outcomes. Successful transfer of that sort suggests that the organism knows which reinforcers were used with which responses and stimuli. In addition, Colwill and Rescorla (1985) have found that reducing the value of a

This research was supported by National Science Foundation Grant BNS 83-08176. Correspondence concerning this article may be addressed to either Robert A. Rescorla, Department of Psychology, 3815 Walnut Street, Philadelphia, PA 19104, or Ruth M. Colwill, Department of Psychology, Brown University, Providence, RI 02912. reinforcer after instrumental training dramatically suppresses responses trained with that reinforcer but leaves unaffected responses trained with other reinforcers. Such reinforcer-specific consequences of devaluation imply an encoding of the reinforcer identity during instrumental training.

Results of this sort have led to the conclusion that organisms form an association between an instrumental response and its outcome. However, theoretical accounts have differed importantly in the ways in which they describe this association as forming and functioning. Two alternatives have received particular attention.

Perhaps the most straightforward possibility was favored by Konorski (1948) and Tolman (1933) and has recently been entertained by Bolles (1972), by Mackintosh and Dickinson (1979), and by Colwill and Rescorla (1986). According to this view, the organism learns about the contingency that the experimenter arranges between the response and the reinforcer; that knowledge is represented as an $\mathrm{R}-\mathrm{O}$ association.

A somewhat more elaborate alternative is envisaged by classical two-process theories (e.g., Rescorla \& Solomon, 1967; Spence, 1956; Trapold \& Overmier, 1972). According to these theories, arranging an instrumental contingency also results in the reinforcer's bearing a Pavlovian relation to the discriminative stimulus. As a result of that relation, an $\mathrm{S}-\mathrm{O}$ association is formed. One consequence of this association is that $\mathrm{S}$ elicits an anticipatory representation of the outcome (historically termed the $r_{g}$ ) that comes to precede the instrumental response. That precedence has been thought by some to allow the stimulus consequences of the $r_{8}$ to become associated with the response (Trapold \& Overmier, 1972). In this way, the animal forms an association in which the anticipation of a particular outcome is the antecedent of the response. One might term this an $\mathrm{O}-\mathrm{R}$ association, to contrast 
it with the $\mathrm{R}-\mathrm{O}$ association described by Konorski and Tolman.

Each of these theoretical views can provide an account of the available data pointing to an encoding of the reinforcer. The two-process theory explains the differential outcomes and transfer results by appealing to the mediating role of the $\mathrm{O}-\mathrm{R}$ association. As a result of discriminative training, the animal forms both $\mathrm{S}-\mathrm{O}$ and $\mathrm{O}-\mathrm{R}$ associations. When different outcomes are used to reinforce different responses in the presence of different stimuli, these associations can provide a route for $S$ to produce a unique $\mathrm{R}$. When the same outcome is used to reinforce all $\mathrm{S} / \mathrm{R}$ combinations, the associations with $\mathrm{O}$ do not allow an $S$ to select a particular $R$. Thus, the two-process theory naturally explains the superiority of learning with differential outcomes and the transfer of a discriminative stimulus across responses based on a shared outcome. However, one can give a similar account of the same data by appealing not to an $\mathrm{O}-\mathrm{R}$ but instead to an $\mathrm{R}-\mathrm{O}$ association, if one simply assumes that the animal is capable of using the R-O association in the "backward" direction (e.g., see Asratyan, 1974).

The adverse effects of posttraining devaluation of the reinforcer can also be explained by both theories. As Colwill and Rescorla (1986) point out, if the organism is viewed as normally employing either an $\mathrm{R}-\mathrm{O}$ or an $\mathrm{O}-\mathrm{R}$ association to generate the response, it is not surprising that devaluation of $\mathrm{O}$ should undermine performance.

However, it is worth noting that some of the devaluation experiments have been conducted in such a way as to reduce the possibility of differential $\mathrm{O}-\mathrm{R}$ associations developing during training. For instance, in one experiment, Colwill and Rescorla (1986) conducted free operant training in which the same manipulandum was followed by one outcome when displaced in one direction and another outcome when displaced in the opposite direction. Subisequent devaluation of one of the outcomes selectively reduced the likelihood of displacement in the direction that had previously produced that outcome. Such an instrumental training procedure, in which both outcomes occur in the presence of the same stimulus environment, makes it relatively difficult for the two-process theory to identify a stimulus that might selectively bring the appropriate reinforcer anticipation forward in time to be associated with one, rather than the other, response.

The experiments reported here were conducted in the attempt to provide further evidence regarding the possible contributions of putative $\mathrm{R}-\mathrm{O}$ and $\mathrm{O}-\mathrm{R}$ associations to instrumental performance. They were particularly directed at assessing the role of these associations in accounting for the transfer and devaluation effects previously reported by Colwill and Rescorla $(1985,1988)$. The technique employed was to arrange for the anticipation of one reinforcer (O1) to precede a response but the occurrence of another reinforcer $(\mathrm{O} 2)$ to follow that same response. This was accomplished by arranging for $\mathrm{R}$ to receive all of its pairings with $\mathrm{O} 2$ in the presence of a stimulus known to elicit an anticipation of O1. According to a two-process theory, this should result primarily in O1-R associations; according to the response-outcome view, it should produce $\mathrm{R}-\mathrm{O} 2$ associations. The relative importance of these two associations was assessed in both the devaluation and the transfer paradigm. In Experiments 1, 2, and 3, a devaluation procedure was used, in which either $\mathrm{O} 1$ or $\mathrm{O} 2$ was followed by a lithium chloride $(\mathrm{LiCl})$ toxin. The question is whether poisoning of $\mathrm{O} 1$ or of $\mathrm{O} 2$ would have a greater adverse effect on the subsequent occurrence of R. In Experiment 4, a transfer procedure was used, to assess whether the instrumental $R$ would be more successfully elicited by a discriminative stimulus associated with $\mathrm{O} 1$ or with $\mathrm{O} 2$.

\section{EXPERIMENT 1}

In this experiment, rats were reinforced with one outcome at a time when they anticipated a different outcome. The manner in which this was accomplished is schematized in Figure 1. Initially, all animals received instrumental discrimination training in which a common response, $\mathrm{R}_{\mathrm{C}}$ (nose poking), was reinforced with one outcome, $\mathrm{O} 1$ (sucrose or pellets) in the presence of one stimulus, S1 (a light or noise); that same response was reinforced with the other outcome, $\mathrm{O} 2$, in the presence of the other stimulus, $\mathbf{S} 2$. This training has been previously shown to endow the stimulus with the ability to evoke differential anticipations of the outcomes (see Colwill \& Rescorla, 1988). Then two new instrumental responses, $R 1$ and $R 2$ (leverpressing and chain pulling), were reinforced, one in the presence of each of the stimuli. For one group of animals (Group Same), the reinforcers delivered during the stimuli were the same as those previously earned in their presence by the nose-poke response. For a second group of animals (Group Switched), the outcomes used in the presence of the stimuli were switched from those employed during nose-poke training. In this way, each discriminative stimulus evoked the anticipation of a unique outcome as a result of nose-poke training; but the new responses were followed by a different outcome in the presence of that anticipation. Following this training, all animals had either $\mathrm{O} 1$ or $\mathrm{O} 2$ devalued by pairing with $\mathrm{LiCl}$. Finally, they were given an extinction choice test between R1 and R2 (chain pulling and leverpressing).

\begin{tabular}{|c|c|c|c|c|}
\hline \multirow{3}{*}{$\begin{array}{l}\text { Group } \\
\text { Same }\end{array}$} & \multicolumn{3}{|c|}{ Design of Experiment 1} & \multirow{3}{*}{$\begin{array}{c}\text { test } \\
R_{1} \text { vs } R_{2}\end{array}$} \\
\hline & 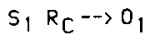 & $S_{1} R_{1} \rightarrow 0_{1}$ & $0_{1}+, 0_{2-}^{-}$ & \\
\hline & $\mathrm{S}_{2} \mathrm{R}_{\mathrm{C}} \longrightarrow \mathrm{O}_{2}$ & $S_{2} R_{2} \rightarrow a_{2}$ & $\mathrm{O}_{2}+\mathrm{O}_{1}-$ & \\
\hline Group & $s_{1} R_{C} \rightarrow 0_{1}$ & $S_{1} R_{1} \cdots O_{2}$ & $\mathrm{O}_{1}+, \mathrm{O}_{2}^{-}$ & test \\
\hline Switched & $S_{2} R_{C} \rightarrow O_{2}$ & $S_{2} R_{2} \rightarrow 0_{1}$ & $\mathrm{O}_{2}+, \mathrm{O}_{1}^{-}$ & $R_{1}$ vs \\
\hline
\end{tabular}

Figure 1. Design of Experiment 1. (R, $\mathbf{R}_{\mathrm{r}}$, and $\mathbf{R 2}$ are instrumental responses-nose poking, leverpressing, and chain pulling; S1 and $S 2$ are discriminative stimuli-noise and light; $O 1$ and $O 2$ are reinforcers-sucrose and pellets.) 
The two theories make a common prediction for Group Same. Both anticipate that devaluation of an outcome should selectively depress the response (either leverpress or chain pull) that it trained. In that group, the anticipated outcome that the two-process theory sees as entering into an $\mathrm{O}-\mathrm{R}$ association is the same as the actual outcome that the other theory sees as entering into an $\mathrm{R}-\mathrm{O}$ association. However, the theories differ in their predictions for Group Switched. According to the two-process theory, following a response with $\mathrm{O} 2$ in the presence of the anticipation of $\mathrm{O} 1$ should establish an O1-R association; according to the response-reinforcer view, it should lead to an $\mathrm{R}-\mathrm{O} 2$ association. Consequently, the two-process theory expects $R$ to be most affected by devaluing the outcome anticipated $(\mathrm{O} 1)$, whereas the response-reinforcer account expects $\mathrm{R}$ to be most affected by devaluing the outcome that followed it $(\mathrm{O} 2)$.

A subsequent test was given in order to verify that the original discrimination training with the nose-poke response had indeed endowed the stimuli with the ability to evoke different anticipations that persisted throughout training of leverpressing and chain pulling. During this test, the animals were given the opportunity to nose poke in the presence of those stimuli. To the degree that the stimuli evoked anticipations of different outcomes, devaluation of one outcome should have selectively depressed responding during its associated stimulus.

\section{Method}

\section{Subjects}

The subjects were 16 experimentally naive Sprague-Dawley male rats (Holtzman $\mathrm{Co}$.) about $\mathbf{1 0 0}$ days old at the start of the experiment. They were housed in individual cages and maintained at $80 \%$ of their free-feeding weights. Water was available on an ad-lib schedule.

\section{Apparatus}

The apparatus consisted of eight identical Skinner boxes measuring $22.9 \times 20.3 \times 20.3 \mathrm{~cm}$. The two end walls of the chamber were aluminum; the sidewalls and the ceiling were clear Plexiglas. Each chamber had a recessed food magazine in the center of one end wall. A small metal cup measuring $1.25 \mathrm{~cm}$ in diameter and $1.5 \mathrm{~cm}$ deep was sunk in the floor of each food magazine. To the left of the magazine was a lever, and to the right was a chain suspended from a relay mounted on the roof of the chamber. Located directly above the magazine was a nose-poke manipulandum, which consisted of an aperture, $2 \mathrm{~cm}$ wide and $1.3 \mathrm{~cm}$ deep. The back of this aperture was covered by a metal plate that operated a relay switch whenever it was depressed. Access to these manipulanda could be prevented by covering the lever with a metal plate, by retracting the chain through an opening in the ceiling, and by inserting a jeweled light cover into the aperture of the nose-poke manipulandum. The floor of the chamber was composed of $0.48-\mathrm{cm}$ stainless steel rods spaced $1.9 \mathrm{~cm}$ apart. Each Skinner box was enclosed in a sound- and light-resistant shell. Mounted on the inside wall of each shell was a speaker that permitted presentation of a white noise $(\mathrm{N})$, measuring approximately $76 \mathrm{~dB}$ re $20 \mu \mathrm{N} / \mathrm{m}$, that served as one discriminative stimulus. Next to the speaker was a 6-W light (L), the illumination of which served as a second stimulus. An additional speaker allowed presentation of an $1800-\mathrm{Hz}$ tone (T), measuring approximately $76 \mathrm{~dB}$ re $20 \mu \mathrm{N} / \mathrm{m}$, that served to signal sucrose reinforcement. The outside roof of the shell supported a solenoid-operated gravity-feed system, which was connected via plastic tubing to the cup in the food magazine. Experimental events were controlled and recorded automatically by relays and microprocessors located in an adjoining room.

\section{Procedure}

All subjects received one session of magazine training consisting of the delivery of $1045-\mathrm{mg}$ food pellets (Formula A: P. J. Noyes Co.) followed by 10 presentations of $0.3 \mathrm{ml} \mathrm{of} 8 \%$ sucrose liquid on a variable-time (VT) 60 -sec schedule. In this and all subsequent phases, the delivery of a pellet was signaled by a distinct click of the pellet dispenser; delivery of sucrose was accompanied by a brief $1800-\mathrm{Hz}$ tone. During this phase, the response manipulanda were not accessible to the animals.

Following magazine training, all subjects received training with the nose-poke manipulandum. In the first session, each nose-poke response was reinforced with pellets; in the second session, each response was reinforced with sucrose. Both sessions terminated after 25 reinforcers had been earned. Nose poking was then reinforced for two 20-min sessions on a variable-interval (VI) 30-sec schedule. Pellets served as the reinforcer in the first of these sessions, and sucrose in the second.

Discriminative stimulus training. Each session of discriminative stimulus training contained 32 presentations of either a $30-\mathrm{sec}$ noise or a 30 -sec light stimulus. Nose-poke responding during a stımulus was reinforced on a VI 30-sec schedule. For half the animals, nose poking was reinforced with pellets in the presence of the light stimulus and with sucrose in the presence of the noise stimulus; for the remaining subjects, pellets reinforced responding during the noise stimulus and sucrose in the light stimulus. For the first two training sessions with each stimulus, the ITI was set at $15 \mathrm{sec}$. It was increased to $30 \mathrm{sec}$ for the next 4 training sessions with each stimulus. For the next two sessions with each stimulus, it was increased to $60 \mathrm{sec}$ and, finally, to $90 \mathrm{sec}$ for the last two sessions of training with each stimulus. Thus, there were 10 training sessions with each discriminative stimulus.

Target response training. The two target responses, lever and chain, were trained initially with cherry-flavored pellets $(P$. J. Noyes Co.). Initially, each response was programmed to produce a reinforcer until 50 reinforcers had been earned. Leverpressing was trained in the first session and chain pulling in the second. Each response was then reinforced first on a VI 30 -sec schedule for one 20-min session and then on a VI 60-sec schedule for another 20-min session. The purpose of this training was to ensure a reasonable level of responding during the presentations of the stimuli in the next stage.

Discriminative stimulus training with lever and chain. The subjects were given four sessions, two with the lever available and two with the chain. Each session contained eight 30 -sec presentations of one of the two stimuli, with an ITI of $30 \mathrm{sec}$. The identity of the stimulus presented in the sessions with the chain and those with the lever was balanced across animals. Responding during the stimuli was reinforced on a VI 15-sec schedule with either food pellets or sucrose. For half the animals (Group Same), the reinforcer earned during a stimulus was the same as that earned by nose poking in the presence of that stimulus; for the remaining animals (Group Switched), the identities of the reinforcers were reversed.

Aversion training. Flavor-aversion conditioning was carried out in the operant chambers over five 2 -day cycles. On the first day of each cycle, the to-be-conditioned reinforcer was delivered on a VT $60-\mathrm{sec}$ schedule. Delivery of the reinforcer was discontinued after $20 \mathrm{~min}$, or whenever five reinforcers had accumulated in the food magazine. Subjects were removed from the chamber and given a $0.5-\mathrm{ml} / \mathrm{kg}$ intraperitoneal (i.p.) injection of $0.6 \mathrm{M}$ lithium chloride $(\mathrm{LiCl})$ before being returned to their home cages. On the second day of each cycle, the other reinforcer was presented on a VT $60-\mathrm{sec}$ schedule for $20 \mathrm{~min}$. At the end of the session, the subjects were simply returned to their home cages. For half the animals, a flavor aversion was conditioned to the food pellets; for the re- 
maining animals, sucrose was paired with the toxin. Designation of the to-be-conditioned reinforcer was balanced across treatment condition: and the stimulus and response identities. Throughou this phase of the study, the response manipulanda were not acces sible to the subjects. To permit observation of consumption, the doors of the sound-attenuating boxes were left open after the first two conditioning cycles and the room was dimly illuminated with red light.

Extinction testing. All subjects received a single 20 -min test with both lever and chain available. Responding was never reinforced. The differential rates of responding during this test constitute the data of primary interest.

Next, to document the presence of $\mathrm{S}$-reinforcer associations, subjects were given a test session containing four presentations of the $30-\mathrm{sec}$ noise and four of the light stimuli with an ITI of $90 \mathrm{sec}$. Only the nose-poke manipulandum was available in these tests, and responding was never reinforced.

Consumption test. To assess the effectiveness of the aversion training, all subjects were given a consumption test. The subjects were placed in the operant chambers, and after $2 \mathrm{~min}$, a single pellet was delivered. An observer recorded whether or not the reinforcer was consumed within $5 \mathrm{~min}$. Half an hour later, the test was repeated with the sucrose reinforcer. No response manipulanda were accessible to the animals during these tests.

\section{Results and Discussion}

Discriminative stimulus control over nose poking was rapidly acquired. This discrimination was measured in terms of a ratio of the rate of responding during the stimulus divided by the sum of the rate during the stimulus and the rate during the ITI. By the end of training, the mean discrimination ratio for the last two sessions, collapsed across stimulus and reinforcer identity, was 0.89 . There were no significant effects of either stimulus or reinforcer identity on discriminative performance.

Training of the target instrumental responses with cherry pellets proceeded uneventfully. In the last session of VI training, the mean rate of leverpressing was 11.0 responses per minute and that of chain pulling was 10.7 responses per minute. This difference was not significant. During the two sessions in which these responses were reinforced in the presence of the stimuli, no significant differences emerged as a function of whether or not the identity of the reinforcer following the response matched that of the reinforcer associated with the stimulus. For Group Same, in which the response-contingent reinforcer was the same as that earned during original discriminative stimulus training, the mean rates of responding collapsed across the two sessions were 11.9 and 12.3 responses per minute during the stimuli and the ITI, respectively. For Group Switched, in which the responsecontingent reinforcer was different from the one that had been presented during original discriminative stimulus training. the comparable rates of responding were 11.7 and 11.9 responses per minute during the stimuli and the ITI, respectively.

The aversion training was successful as judged by the decline in consumption of the poisoned reinforcer over the course of conditioning. By the final cycle, no subject consumed any of the poisoned reinforcers, but all subjects consumed the nonpoisoned reinforcers. During the consumption test administered after the test sessions, no animal consumed the reinforcer that had been paired with a toxin but all animals ate the nonpoisoned reinforcer.

The data of primary interest concern the rates of responding on the lever and chain during the first extinction test. Figure 2 displays those data, in blocks of $4 \mathrm{~min}$, for the same (left panel) and switched (right panel) groups. Within each panel, responding is separated according to whether the consequent reinforcer had been devalued (filled circles) or not (open circles). The animals in Group Same chose between two responses, one of which (filled circles) had both its anticipated and consequent reinforcer devalued. The animals in Group Switched chose between two responses, one of which had its consequent (but not its anticipated) reinforcer devalued (filled circles) and the other of which had its anticipated (but not its consequent) reinforcer devalued (open circles). It is clear that for both groups, poisoning of the consequent reinforcer resulted in marked reduction in performance of that response. To minimize subject variability for purposes of statistical analysis, the total responses on each manipulandum was expressed as a percentage of the number of responses made on that manipulandum during the final session of VI training with cherry pellets. For Group Same, the mean percentages for the devalued consequent and anticipated reinforcers were $13 \%$ and $36 \%$, respectively; for Group Switched, the comparable means were $17 \%$ and $30 \%$. Comparison of these percentages showed significantly less responding when the outcome had been devalued for both Group Same [Wilcoxon $T(8)=3, p<.05$ ] and Group Switched $[T(7)=0, p<.02]$. The magnitude of the devaluation effect was not reliably different between the two groups [Mann-Whitney $U(8,7)=21.5, p>.10]$.

The results for Group Same confirm earlier findings (e.g., those of Colwill \& Rescorla, 1986) that devaluation of a reinforcer selectively depresses responses that earned that reinforcer, even when training takes place in the presence of a stimulus. However, the performance of Group Switched during this test suggests that it is the devaluation of the consequent, rather than the anticipated reinforcer, that has the more profound effect on instrumental responding. Such a result is precisely the one predicted by an R-O theory of instrumental learning. It is inconsistent with the predictions of an $\mathrm{O}-\mathrm{R}$ theory.

However, in order to draw this conclusion, it is essential to demonstrate that the original $\mathrm{S}-\mathrm{O}$ associations persisted through the training of the target responses. Although there is no reason to expect those associations to be disrupted in Group Same, it is quite possible that they were altered during the training of target responses in Group Switched. The training of the target responses was kept brief precisely to minimize the possibility of such a change. Moreover, the results of testing the discriminative stimuli with the nose-poke response provided confirmation that this training had not degraded the original S-O associations. In each group, nose-poke responding was depressed during the stimulus that had originally signaled the now devalued reinforcer. For Group Switched, the 
GROUP SAME

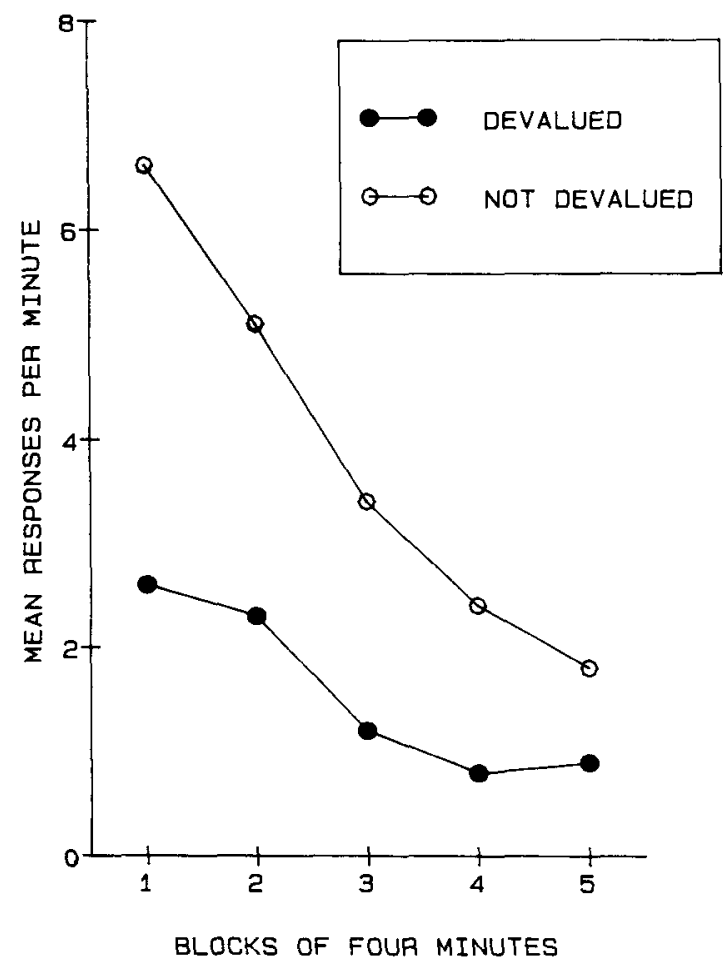

GROUP SWITCHED

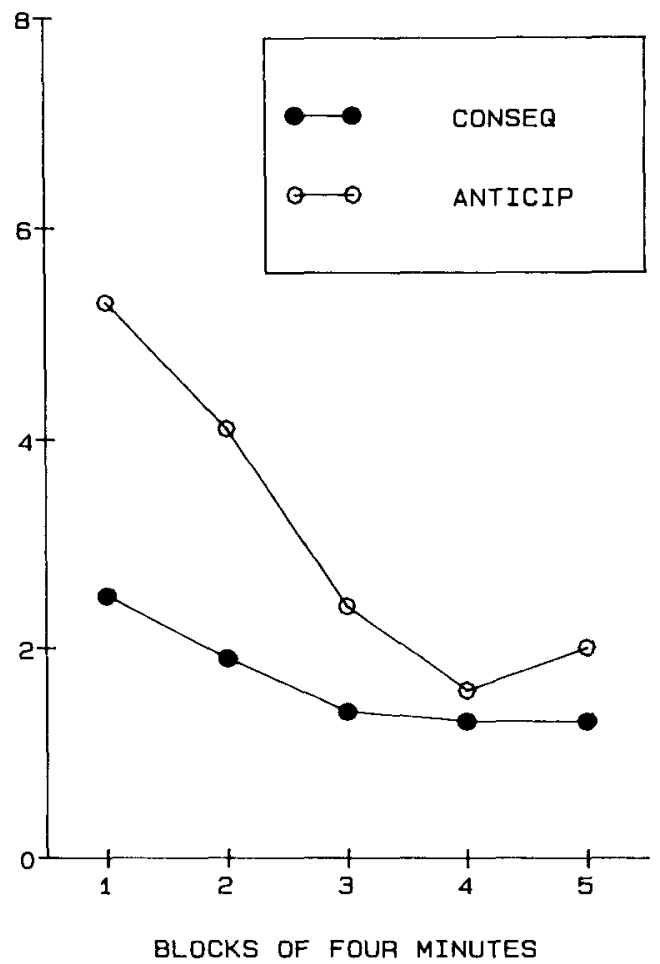

Figure 2. Test results of Experiment 1. Mean responses per minute during the choice extinction test for Group Same (left panel) and Group Switched (right panel). For Group Same, responding is shown separately when the consequent reinforcer was devalued (filled circles) or not (open circles). For Group Switched, responding is shown separately when the CONSEQuent reinforcer was devalued (filled circles) and when the ANTICIPated reinforcer was devalued (open circles).

mean rates of responding for the first and second halves of the test session were 12.6 and 3.0 responses per minute during the stimulus associated with the devalued reinforcer, and 16.3 and 13.9 responses per minute in the presence of the nondevalued stimulus. For Group Same, the comparable response rates were 9.8 and 2.1 responses per minute during the stimulus trained with the devalued reinforcer, and 12.8 and 5.9 responses per minute during the stimulus associated with the intact reinforcer. Analysis of these data revealed that there was significantly less nose poking during the devalued stimulus relative to the nondevalued stimulus in the second half of the test session $[T(14)=12.5, p<.05]$. It is of special importance to note that the ability of a stimulus to evoke the anticipation of its original outcome had been preserved during the training of the target responses with different reinforcers in Group Switched $[T(8)=1$, $p<.01]$.

It seems likely that the continued ability of the stimuli to evoke their original outcomes depends on the brevity of the period over which the target responses were followed by the differential outcomes in the presence of those stimuli. One consequence of using such a brief period of differential reinforcement is that the stimuli showed no evidence of developing stimulus control over the target responses. It is important to note, however, that such stimulus control is not relevant to the intention in the present experiments. The goal is to identify the nature of the association between $\mathrm{R}$ and $\mathrm{O}$ that is responsible for the impact of devaluation of $\mathrm{O}$ upon responding. In this experiment, such a devaluation effect was found after training conditions that provided an opportunity for both $\mathrm{O}-\mathrm{R}$ and $\mathrm{R}-\mathrm{O}$ associations. The role of the discriminative stimulus was to guarantee the presence of the expectation of one outcome at a time when the response was followed by a different outcome. It is clear that the discriminative stimulus played that role whether or not it developed discriminative control itself. Of course, with additional training, the stimulus would develop discriminative control. However, under those conditions, we could not maintain its ability to elicit the original expectations, and therefore could not discriminate between the contributions of the $\mathrm{R}-\mathrm{O}$ and $\mathrm{O}-\mathrm{R}$ associations. It might even be that with extensive training, the expectations elicited by the stimulus would develop control over responding. Hence the contribution of $\mathrm{O}-\mathrm{R}$ associations might develop with more substantial training. But it is evident that such $\mathrm{O}-\mathrm{R}$ associations are not as powerful as are $\mathrm{R}-\mathrm{O}$ associations in the initial encoding of the reinforcer, as revealed by the devaluation procedure. 
Consequently, this experiment shows that performance of an instrumental response that has been followed by one reinforcer in the presence of an expectation of a different reinforcer is more profoundly affected by degrading the value of the consequent outcome than of the anticipated outcome. This suggests that a primary way in which an outcome becomes associated with the response occurs in the form of an $\mathrm{R}-\mathrm{O}$ association. Certainly the contribution of the $\mathrm{R}-\mathrm{O}$ association appears more substantial than that of an $O-R$ association, at least in initial learning.

The next two experiments were designed to provide further evaluation of the role of $O-R$ associations in instrumental behavior. These studies used what might be thought to be more sensitive assessments intended to maximize detection of any contribution of O-R associations to behavior. In Experiment 2, a procedure similar to that of Experiment 1 was used, except that the test conditions involved the presentation of stimuli known to elicit expectations of the anticipated outcome. The question was whether presenting strong anticipations of the outcomes during the test would reveal any contribution of $O-R$ associations. Experiment 3 addressed the possibility that the presence of differential $\mathrm{R}-\mathrm{O}$ associations had masked detection of the $\mathrm{O}-\mathrm{R}$ associations in Experiment 1. Thus, instrumental behaviors were established with common consequences but unique anticipated outcomes. By devaluing one of the anticipated outcomes and measuring choice, a pure assessment of the control over responding by the $\mathrm{O}-\mathrm{R}$ association was permitted.

\section{EXPERIMENT 2}

The critical test between the two target responses in Experiment 1 took place in the absence of any explicit stimuli that would have evoked strong representations of the anticipated outcomes. In order to provide a generally applicable account of encoding of the reinforcer, two-process theory must presume that such anticipations are present by virtue of background conditioning. Hence the test procedure used in Experiment 1 constitutes an appropriate way to evaluate the contribution of $\mathrm{O}-\mathrm{R}$ associations within the framework of a two-process theory. Nevertheless, it is possible that had explicit stimuli known to elicit those anticipations been present, their control over responding through $\mathrm{O}-\mathrm{R}$ associations would have been detected. Thus, the animals in Experiment 2 received a training history identical to those in Group Switched of Experiment 1 . They differed only in their treatment during testing. In Experiment 2, assessment of the relative degree of control by anticipated and consequent outcomes was made in the presence of the discriminative stimuli during the test with the target responses.

All animals received initial instrumental discrimination training identical to that in Experiment 1. A common response was reinforced with pellets in the presence of one discriminative stimulus and with sucrose in the presence of another discriminative stimulus. The next treatment phase was the same as that given to Group
Switched. Two new responses were trained, one in the presence of each discriminative stimulus. The outcomes used in the presence of the stimuli were switched from those employed during initial training. Thus, each target response was followed by one outcome in the presence of an anticipation of a different outcome evoked by the discriminative stimulus. Following this training, all animals had one of the outcomes devalued by pairing with $\mathrm{LiCl}$. Finally, they were given an extinction choice test between the two target responses, with occasional compound presentations of the two discriminative stimuli. Compound, rather than separate, discriminative stimulus presentations were used because the two stimuli were associated with outcomes that had been differentially devalued, and hence would differentially interfere with responding for that reason.

According to two-process theory, following a response with one outcome $(\mathrm{O} 2)$ in the presence of the anticipation of a different outcome (O1) should establish an O1-R association with the result that responding should be undermined most by poisoning of O1. Alternatively, if associations are formed between the response and its consequence, responding should be undermined most by devaluation of $\mathrm{O} 2$. The compound presentation of the two discriminative stimuli guaranteed the presence of the anticipation of both outcomes during the test.

\section{Subjects and Apparatus}

\section{Method}

The subjects were 16 experimentally naive Sprague-Dawley male rats (Holtzman Co.) about 100 days old at the start of the experiment. They were housed and maintained in the same way as subjects in Experiment 1. The apparatus was that used in Experiment 1, except that delivery of the sucrose was accompanied by a $0.5-\mathrm{sec}$ buzzer.

\section{Procedure}

The procedure for magazine training and initial nose-poke training was identical to that described in Experiment 1, with one exception: the subjects were allowed to earn 30 reinforcers in each session of CRF training.

Discriminative stimulus training. The procedure for bringing nose-poke responding under discriminative control was identical to that described in Experiment 1.

Target response training. The two target responses, lever and chain, were pretrained with cherry-flavored pellets (P. J. Noyes Co.), following the procedure of Experiment 1. This treatment ensured that the responses would occur during the stimulus presentations in the next phase.

Discriminative stimulus training with the target responses. This phase was identical to that given to the subjects in Group Switched of Experiment 1. There were four sessions, two with the lever available and two with the chain. Each session contained eight 30-sec presentations of one of the two stimuli, with an ITI of $30 \mathrm{sec}$. The identity of the stimulus presented in the sessions with the chain and those with the lever was balanced across animals. Responding during the stimuli was reinforced on a VI 15-sec schedule. For all subjects, the reinforcer earned during one stimulus was the one scheduled during the other stimulus in original training.

Aversion training. Flavor-aversion conditioning was carried out in the operant chambers over six 2-day cycles. The details of this procedure are the same as those outlined in Experiment 1 . For half 
the animals, the food pellets were paired with the toxin; for the remaining animals sucrose was conditioned. Designation of the tobe-conditioned reinforcer was balanced across stimulus and response identity.

Extinction testing. All subjects received a single 8-min test with both lever and chain available. Responding was never reinforced. During the session, there were eight 30 -sec trials on which the two stimuli were presented simultaneously. The ITI was $30 \mathrm{sec}$. The differential rates of responding in the presence of this stimulus compound constitute the data of primary interest.

To document the presence of S-reinforcer associations, subjects were given a test session containing four presentations each of the $30-\mathrm{sec}$ noise and light stimuli with an ITI of $90 \mathrm{sec}$. Only the nosepoke manipulandum was available in these tests, and responding was never reinforced.

Consumption test. To assess the effectiveness of the aversion training, all subjects were given a consumption test identical to that described in Experiment 1.

\section{Results and Discussion}

Discriminative stimulus control over nose poking was rapidly acquired. By the end of training, the mean discrimination ratio for the last two sessions, collapsed across stimulus and reinforcer identity, was 0.81 . There was no significant effect of reinforcer identity, but discriminative control by the light (mean discrimination ratio was 0.84 ) was slightly better than that by the noise (mean discrimination ratio was 0.78 ): $T(16)=21, p<.05$.

ITI



Training of the target instrumental responses with cherry pellets proceeded smoothly. In the last session of VI training, the mean rate of leverpressing was 10.4 responses per minute and that of chain pulling was 10.5 responses per minute. During the two sessions in which these responses were reinforced in the presence of the stimuli, there were no significant differences in the rates of responding as a function of stimulus identity. The mean rate of responding during the discriminative stimuli for the two sessions combined was 9.7 responses per minute; the mean response rate during the ITI was 10.1 responses per minute. The difference between these rates was not significant.

The aversion training was successful as judged by the decline in consumption of the poisoned reinforcer over the course of conditioning. By the final cycle, no subject consumed any of the poisoned reinforcers, but all subjects consumed the nonpoisoned reinforcers. During the consumption test administered after the test sessions, no animal consumed the reinforcer that had been paired with a toxin, but all animals ate the nonpoisoned reinforcer.

The data of primary interest concern the rates of responding on the lever and chain during the first extinction test with the discriminative stimulus compound. Figure 3 displays those data in blocks of two trials. Responding during the ITI is shown on the left panel, and responding dur-

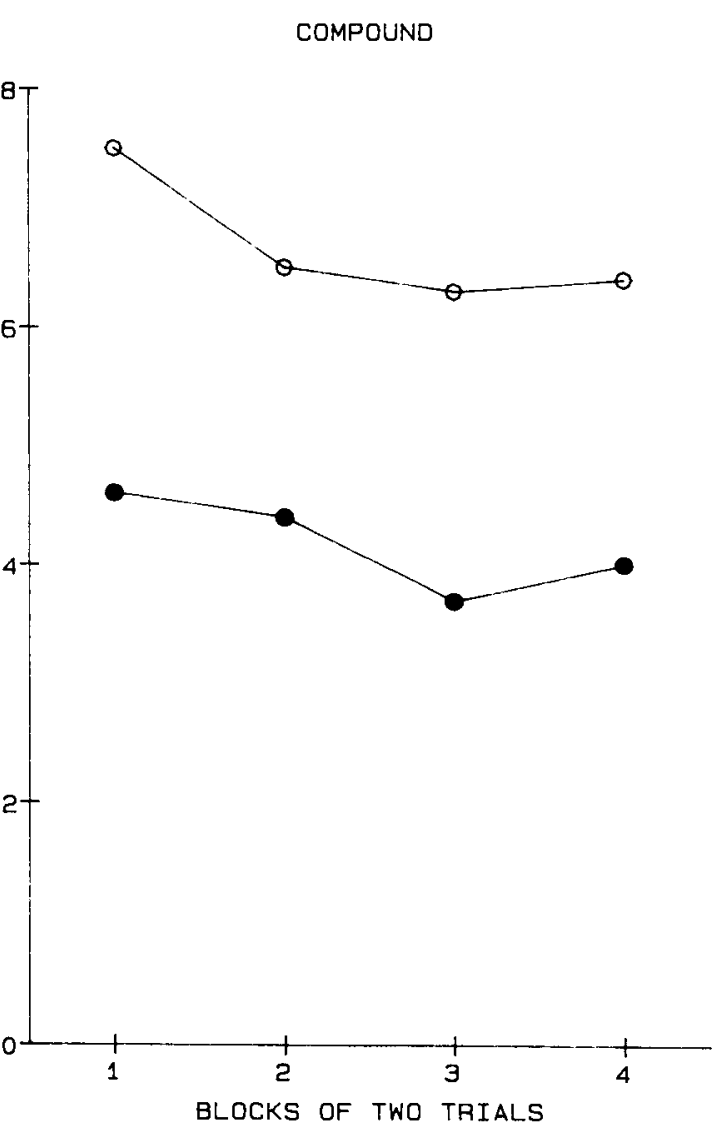

Figure 3. Test results of Experiment 2. Mean responses per minute in the choice extinction test during the ITI (left panel) and during the stimulus compound (right panel). In each panel, response rates are shown separately when the CONSEQuent reinforcer was devalued (filled circles) and when the ANTICIPated reinforcer was devalued (open circles). 
ing the stimulus compound is shown on the right. Within each panel, responding is presented separately according to whether the anticipated reinforcer had been devalued (open circles) or the consequent reinforcer had been devalued (filled circles). It is clear that poisoning of the consequent reinforcer resulted in greater reduction in performance of that response during the ITI period $[T(15)=23$, $p<.05]$. That result replicates the results of Group Switched from Experiment 1.

The right panel of Figure 3 shows responding during the compound consisting of the two discriminative stimuli. The data pattern is quite similar to that observed for responding in the ITI. Poisoning of the consequent reinforcer depressed instrumental responding in the presence of the stimulus compound more than did poisoning of the anticipated reinforcer $[T(16)=30, p=.05]$. Even when the compound stimulus guaranteed that the expectation of the anticipated reinforcer would be present, the value of the consequent reinforcer was more important in determining performance.

It is also of interest to note that the compound stimulus produced an overall enhancement of responding. For the session as a whole, responding was greater than that during the ITI, whether the consequent $[T(16)=24.5$, $p<.05]$ or the anticipated $[T(15)=18.5, p<.05]$ reinforcer had been devalued. It is possible that this increase in responding is attributable to a general augmenting power of discriminative stimuli. But previous results (Colwill $\&$ Rescorla, 1988) suggest that it depends on whether or not the discriminative stimuli and the responses have a history of training with a common outcome. That possibility is exploited further in Experiment 4.

The results of this experiment reinforce the conclusion of Experiment 1 that it is the devaluation of the consequent, rather than the anticipated, reinforcer that has the more profound effect on instrumental responding. It seems clear that even under circumstances that should promote detection of the effect of $\mathrm{O}-\mathrm{R}$ associations, it is $\mathrm{R}-\mathrm{O}$ associations that are more influential in governing performance.

However, as was argued in Experiment 1, in order to draw this conclusion, it is important to demonstrate that the original S-O associations persisted through the training of the target responses. The results of testing the discriminative stimuli with the nose-poke response provided confirmation that the original S-O associations had survived. The mean rate of nose-poke responding during the test session was 3.1 responses per minute during the stimulus associated with the devalued reinforcer and 6.2 responses per minute in the presence of the nondevalued stimulus. Analysis of these data revealed that there was significantly less nose poking during the devalued stimulus relative to the nondevalued stimulus $T(14)=15$, $p<.05]$. This result provides strong confirmation that the ability of a stimulus to evoke the anticipation of its original outcome is preserved during the training of the target responses.

Consequently, Experiment 2 confirms the results of Experiment 1 . Even in the presence of stimuli known to elicit anticipations of the outcomes, the $\mathrm{R}-\mathrm{O}$ association proved a more potent controller of behavior than did the $\mathrm{O}-\mathrm{R}$ association.

\section{EXPERIMENT 3}

Although Experiments 1 and 2 suggest that $\mathrm{R}-\mathrm{O}$ associations are more important than $\mathrm{O}-\mathrm{R}$ associations in controlling behavior, they leave open the possibility that $\mathrm{O}-\mathrm{R}$ associations make some contribution early in training. There is the suggestion in Figure 2 that the devaluation effect was larger in Group Same than in Group Switched. Although such an outcome could indicate a contribution of an O-R association, it failed to reach statistical significance. Experiment 3 was designed to examine in more detail the possibility that $\mathrm{O}-\mathrm{R}$ associations might influence early instrumental performance.

By arranging for each $R$ to have one outcome as its antecedent and the other as its consequent, the design of Experiments 1 and 2 pitted the $\mathrm{O} 1-\mathrm{R}$ and $\mathrm{R}-\mathrm{O} 2$ associations against each other. It seems possible that the strong impact that devaluing $\mathrm{O} 2$ had upon responding partially masked the presence of an O1-R association. Consequently, in Experiment 3, each of two responses was arranged to have a unique anticipation of either $\mathrm{O} 1$ or $\mathrm{O} 2$ as an antecedent, but both responses were followed by a common third outcome. Then either $\mathrm{O} 1$ or $\mathrm{O} 2$ was devalued and the impact of that devaluation on responding was inspected. The responses thus differed only in the possibility of having different $\mathrm{O}-\mathrm{R}$ associations.

The subjects were initially trained to nose poke for sucrose in the presence of one stimulus and for pellets during a different stimulus. This training was intended to establish $\mathrm{S} 1-\mathrm{O} 1$ and $\mathrm{S} 2-\mathrm{O} 2$ associations. Then two new instrumental responses, leverpress and chain pull, were reinforced with cherry pellets, one (R1) in the presence of $\mathrm{S} 1$ and one (R2) in the presence of S2. This training should have allowed for the formation of O1-R1 and $\mathrm{O} 2-\mathrm{R} 2$ associations. Finally, either $\mathrm{O} 1$ or $\mathrm{O} 2$ was devalued by pairing with a $\mathrm{LiCl}$ toxin. If expectations of $\mathrm{O} 1$ or $\mathrm{O} 2$ had acquired discriminative control over instrumental responding, then the subsequent poisoning of either $\mathrm{O} 1$ or $\mathrm{O} 2$ ought selectively to have depressed performance of the response associated with the expectation of the devalued outcome.

\section{Subjects and Apparatus}

\section{Method}

The subjects were 32 experimentally naive Sprague-Dawley male rats (Holtzman Co.) about 100 days old at the start of the experiment. They were housed and maintained in the same way as subjects in Experiment 1. The apparatus was the same as that used in Experiment 1 . In order to maximize detection of any effect, a total of 32 animals were run in two replications $(N=16$ in each replication). The data were combined for this presentation.

\section{Procedure}

The procedure for magazine training and initial nose-poke training was identical to that described in Experiment 1. 
Discriminative stimulus training. The protocol for establishing discriminative stimulus control over nose poking was identical to that used in Experiment 1. Thus, half the animals were trained to nose poke for pellets in the presence of the light and to nose poke for sucrose in the presence of the noise. For the remaining animals, responding earned sucrose during the light stimulus and pellets during the noise stimulus. As in Experiment 1, there were 10 training sessions with each discriminative stimulus.

Target response training. The two target responses, lever and chain, were trained with cherry-flavored pellets (P. J. Noyes Co.), following the procedure described in Experiment 1. As in that study, the purpose of this training was to ensure a reasonable level of target responding during the presentations of the stimuli in the next stage.

Discriminative stimulus training with lever and chain. The subjects were given four sessions, two with the lever available and two with the chain. Each session contained eight 30 -sec presentations of one of the two stimuli with an ITI of $30 \mathrm{sec}$. The identity of the stimulus presented in the sessions with the chain and those with the lever was balanced across animals. Responding during the stimuli was reinforced on a VI 15-sec schedule with cherry-flavored food pellets.

Aversion training. Flavor-aversion conditioning was identical to that of Experiment 1 but was carried out over six 2-day cycles. For half the animals, a flavor aversion was conditioned to the food pellets; for the remaining animals, sucrose was poisoned. Designation of the to-be-conditioned reinforcer was balanced across stimulus and response identity.

Extinction testing. All subjects received, on each of 2 consecutive days, a single 20-min test session with both lever and chain available. Responding was never reinforced.

To document the presence of S-reinforcer associations, subjects were given a test session containing four presentations each of the $30-\mathrm{sec}$ noise and light stimuli with an ITI of $90 \mathrm{sec}$. Only the nosepoke manipulandum was available in this test, and responding was never reinforced.
Consumption test. To assess the effectiveness of the aversion training, all subjects were given a consumption test. The details of this procedure are the same as those described in Experiment 1.

\section{Results and Discussion}

Discriminative stimulus control over nose poking was rapidly acquired. By the end of training, the mean discrimination ratio for the last two sessions, collapsed across stimulus and reinforcer identity, was 0.87 . There were no significant effects of either stimulus or reinforcer identity on discriminative performance.

Training of the target instrumental responses with cherry pellets proceeded smoothly. In the last session of VI training, the mean rates of leverpressing and chain pulling were 11.6 and 12.4 responses per minute, respectively. This difference was not significant. During the two sessions in which these responses were reinforced in the presence of the stimuli, the average rate of responding was 15.5 responses per minute during the stimuli and 14.7 responses per minute during the ITI.

The aversion training was successful, as judged by the decline in consumption of the poisoned reinforcer over the course of conditioning. By the final cycle, no subject consumed any of the poisoned reinforcers, but all subjects consumed the nonpoisoned reinforcers. The final consumption test yielded an identical data pattern.

The results of primary interest are the rates of responding on the lever and chain during the two extinction tests. Figure 4 displays those data plotted in blocks of 4 min. Responding is shown separately, as a function of whether the reinforcer associated with the stimulus in whose

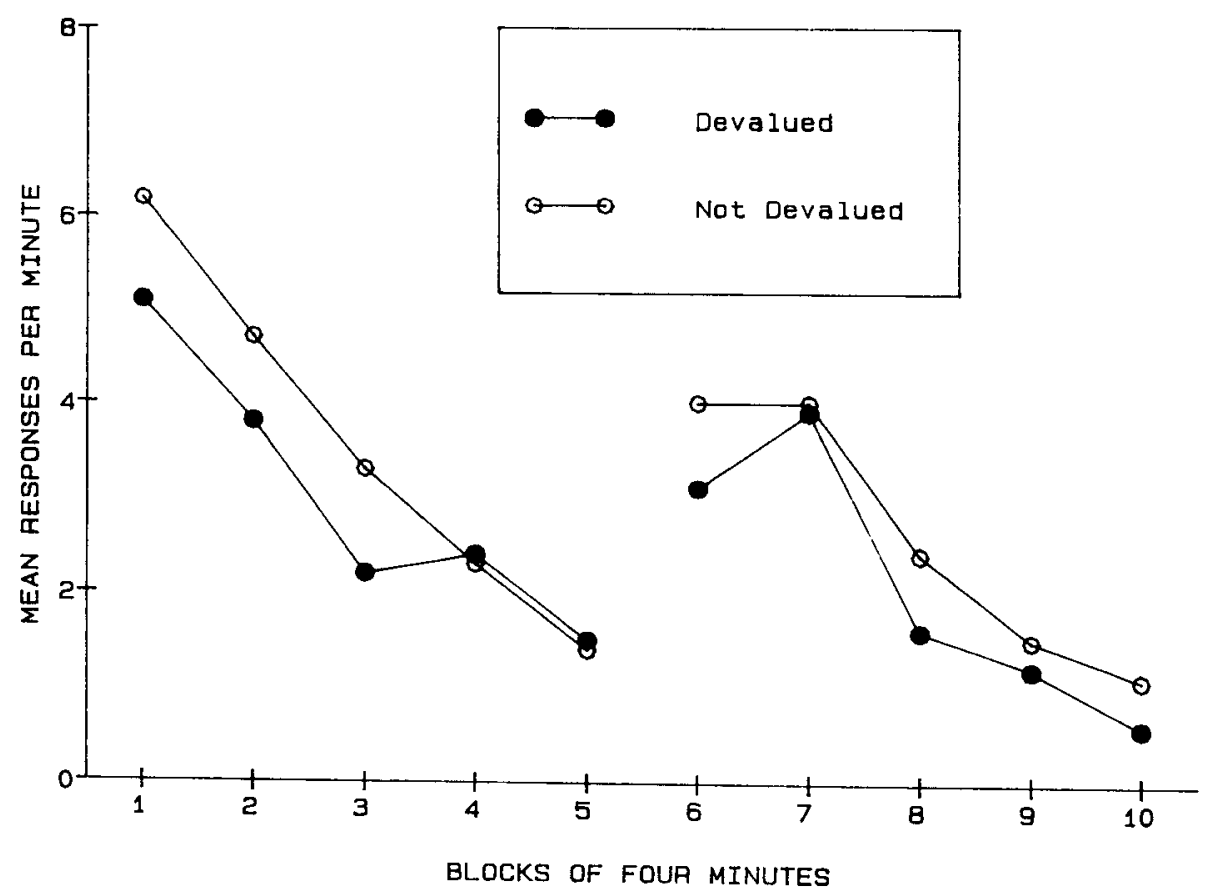

Figure 4. Test results of Experiment 3. Mean responses per minute in the two extinction tests are shown separately when the anticipated reinforcer was devalued (filled circles) or not (open circles). 
presence the response had been reinforced had been devalued (filled circles) or not (open circles). Although there appears to be a very slight reduction in performance of the response whose stimulus was associated with a devalued reinforcer, that difference did not prove to be statistically reliable. A Wilcoxon test performed on the response rates expressed as a percentage of those in the final VI sessions did not approach statistical reliability for either the first $[T(29)=156, z=1.33]$ or second test session $[T(31)=168.5, z=1.56]$ or for the two sessions combined $[T(31)=178, z=1.37]$. The mean percentage response rate for the devalued reinforcer was $24.9 \%$ in the first test session and $17.5 \%$ in the second test session; the comparable means for the nondevalued reinforcer were $29.9 \%$ and $22.8 \%$. Comparable analyses on the absolute rates of responding also failed to reveal any significant differences.

The results of the test session in which the two stimuli were presented with their original instrumental response available verified that the original S-O associations were still intact. The mean rate of responding during the stimulus whose reinforcer had been paired with a toxin $(6.2$ responses per minute) was considerably lower than that during the stimulus whose reinforcer was not devalued ( 8.6 responses per minute): $T(30)=123, p<.05$. That difference indicates that the training of the lever and chain responses with cherry pellets did not destroy the original $\mathrm{S}$-reinforcer associations. Thus, it is clear that the stimuli had continued to evoke differential expectations throughout the training of the target responses.

The results of Experiment 3 provide little evidence that the expectation of a reinforcer exerts control over performance of an instrumental response trained in the presence of that expectation. This conclusion is based on the failure of responding to be significantly altered by a subsequent change in the value of the anticipated reinforcer. That outcome was not due to the lack of opportunity for the reinforcer expectation to gain control over the instrumental response. The preservation of the original S-reinforcer associations indicates the availability of these reinforcer expectations to serve as discriminative stimuli during the training of the target responses. Of course, further experiments or other detection procedures might reveal a contribution of an $\mathrm{O}-\mathrm{R}$ association; however, our failure to find a reliable effect using a substantial number of animals in a procedure quite sensitive to the contribution of $\mathrm{R}-\mathrm{O}$ associations suggests that $\mathrm{O}-\mathrm{R}$ associations may play a relatively minor role in the initial stages of instrumental learning.

\section{EXPERIMENT 4}

In the previous three experiments, a devaluation procedure was used to evaluate the relative importance of $\mathrm{R}-\mathrm{O}$ and $\mathrm{O}-\mathrm{R}$ associations in instrumental performance. In Experiment 4, a transfer technique was exploited to address the same question. As in Experiment 1, a response earned one outcome $(\mathrm{O} 2)$ in the presence of the anticipation of a different outcome (O1). However, neither $\mathrm{O} 1$ nor $\mathrm{O} 2$ was devalued. Instead, in Experiment 4, the response was tested in the presence of a discriminative stimulus known to be associated with one or the other outcome. Colwill and Rescorla (1988) have reported that discriminative stimuli transfer preferentially to responses trained with the same reinforcer. Consequently, the question of interest is whether that transfer is mediated by the outcome anticipated prior to the response (O1) or that delivered consequent upon the response $(\mathrm{O} 2)$.

The design of this experiment is shown in Figure 5. To establish appropriate $\mathrm{S}-\mathrm{O}$ associations, the subjects were reinforced for responding in the presence of four different stimuli, two visual (S1 and S2) and two auditory (S3 and $\mathbf{S 4})$. Nose-poke responses $\left(\mathrm{R}_{\mathrm{C}}\right)$ produced sucrose $(\mathrm{O} 1)$ during one of the auditory and one of the visual stimuli, and pellets (O2) during the other. Then, leverpressing and chain pulling (R1 and R2) were established with either a pellet or a sucrose outcome; responding on one manipulandum was reinforced during one auditory stimulus and responding on the other during the remaining auditory stimulus. The response-reinforcer combinations were such that the reinforcer used as a consequent was different from the reinforcer that had previously occurred during the stimulus. As a result, two instrumental responses were established, each one with an antecedent reinforcer expectation that was different from the consequent reinforcer. Finally, the subjects were tested with the lever and chain available and with occasional presentations of the two visual stimuli. If responses are associated with their consequences, then the stimulus should select the response whose consequent reinforcer is the same as that associated with the stimulus (e.g., S3 should promote $R 2$ rather than $R 1$ ). On the other hand, if the antecedent to a response controls its performance, then the stimulus should select the response whose antecedent reinforcer expectation is the same as that associated with the stimulus (e.g., S3 should promote R1 rather than $\mathrm{R} 2$ ).

\section{Method}

\section{Subjects and Apparatus}

The subjects were 16 experimentally naive Sprague-Dawley male rats (Holtzman Co.) about 100 days old at the start of the experi-

$$
\begin{aligned}
& \text { Design of Experiment } 4 \\
& \begin{array}{l|l|c}
S_{1} R_{C} \rightarrow O_{1} & \\
S_{2} R_{C}->O_{2} & S_{1} R_{1}->0_{2} & \text { Test } \\
S_{3} R_{1} v S_{2} \\
S_{3} R_{C}->0_{1} & S_{2} R_{2}->0_{1} & S_{4} R_{1} \text { vs } R_{2} \\
S_{4} R_{C}-\rightarrow 0_{2} & &
\end{array}
\end{aligned}
$$

Figure 5. Design of Experiment 4. ( $\mathbf{R}_{\mathrm{c}}, \mathbf{R 1}$, and $\mathrm{R2}$ are instrumental responses-nose poking, leverpressing, and chain pulling; S1, $\mathbf{S 2}, \mathbf{S 3}$, and $\mathrm{S} 4$ are discriminative stimuli-noise, tone, steady light, and flashing light; $\mathrm{O1}$ and $\mathrm{O2}$ are reinforcers-sucrose and pellets.) 
ment. They were housed and maintained in the same way as were the subjects in Experiment 1. The apparatus was that used in Experiment 1, except that delivery of the sucrose was accompanied by a 0.5 -sec buzzer. In addition to the noise and light stimuli used in the previous experiments, an $1800-\mathrm{Hz}$ tone and a flashing light served as discriminative stimuli in this study. The additional 6-W light was mounted close to the grid floor on the outside of the operant chamber on the edge of the wall opposite the magazine. Its rate of interruption was 2.5 per second.

\section{Procedure}

The procedure for magazine training and initial nose-poke training was identical to that described in Experiment 1. However, there were an additional four sessions of nose-poke training, two with pellets serving as the reinforcer and two with sucrose. These sessions were each $20 \mathrm{~min}$ long, and responding was reinforced on a VI 60-sec schedule.

Discriminative stimulus training. All subjects were trained to nose poke in the presence of four different discriminative stimuli. A session consisted of either 16 presentations of each of the two 30 -sec auditory stimuli, noise (N) and tone (T), or an equivalent number of each of the two 30-sec visual stimuli, the steady light $(\mathrm{L})$ and the flashing light (l). Responding during one of the auditory and one of the visual stimuli was reinforced with pellets; responding during the other member of each pair was reinforced with sucrose. The value of the ITI was gradually increased across sessions. For the first 2 sessions with each stimulus modality, the ITI was $15 \mathrm{sec}$; for the next 2 sessions, it was set at $30 \mathrm{sec}$. It was lengthened to $60 \mathrm{sec}$ for the next 4 sessions and finally to $90 \mathrm{sec}$ for the last 4 sessions. Thus, there were 12 sessions containing auditory stimuli and 12 with visual stimuli. Whether a session contained auditory or visual stimuli was determined randomly, with the restriction that there could be no more than 3 consecutive sessions with same modality stimuli.

Target response training. The two target responses, lever and chain, were trained with cherry-flavored pellets (P. J. Noyes Co.), following the procedure described in Experiment 1. As in that study, the purpose of this training was to ensure a reasonable level of target responding during the presentations of the stimuli in the next stage.

Discriminative stimulus training with lever and chain. The subjects were given four sessions, two with the lever available and two with the chain. Each session contained eight $30-\mathrm{sec}$ presentations of one of the two auditory stimuli with an ITI of $30 \mathrm{sec}$. The choice of which auditory stimulus was to be presented in the sessions with the chain and which one with the lever was balanced across animals Responding during the stimuli was reinforced on a VI 15-sec schedule, with whichever reinforcer, pellets or sucrose, that had not been earned by the nose-poke response in the presence of that stimulus. Thus, all animals were treated exactly like the animals in Group Switched of Experiment 1.

Extinction testing. All subjects received, on each of 2 consecutive days, a test session containing 8 presentations each of the two visual stimuli with an ITI of $30 \mathrm{sec}$. Both lever and chain were available, but responding was never reinforced.

Following these tests, the subjects received five additional sessions of discriminative stimulus training with the nose-poke response; the first of these contained the auditory stimuli, and the remaining four contained the visual stimuli. The ITI was always $90 \mathrm{sec}$. The intention of this training was to ensure maintenance of the association between the stimuli and their differential outcomes.

The subjects were then retested for one session with the visual stimuli and the lever and chain responses. The procedure was identical to that for the previous two tests.

Finally, the two auditory stimuli were tested. That session contained eight 30 -sec presentations each of the $\mathrm{N}$ and $\mathrm{T}$ separated by an ITI of $30 \mathrm{sec}$. Both the lever and chain were available, but responding was never reinforced.

\section{Results and Discussion}

Discriminative stimulus control over nose poking was rapidly acquired. By the end of training, the mean discrimination ratio for the final session with the auditory stimuli was 0.89 , and for the visual stimuli, it was 0.91 . There were no significant effects of either stimulus or reinforcer identity on discriminative performance.

Training of the target instrumental responses with cherry pellets proceeded smoothly. In the last session of VI training, the mean rate of leverpressing was 9.9 responses per minute and that of chain pulling was 10.6 responses per minute. This difference was not significant. During the two sessions in which these responses were reinforced in the presence of the stimuli, the average rate of responding was 10.6 responses per minute during the stimuli and 10.7 responses per minute during the ITI.

The results of primary interest concern the rates of responding on the lever and chain during the three extinction tests with intermittent presentations of the visual stimuli. Figure 6 displays those data plotted in blocks of two trials. Responding is shown separately as a function of whether the reinforcer associated with the visual stimulus was the same as that which had served as a consequence of the response (filled circles) or as that which had been anticipated when the response was trained (open circles). Responding is also shown during the ITI in the absence of any stimulus. Within each test session, it is clear that the stimuli preferentially enhanced performance of the response that had the same consequent reinforcer as that previously associated with the stimulus. Comparison of the rates of responding during the stimuli collapsed across the three test sessions revealed significantly more responses for the same-consequent than for the sameanticipation $[T(16)=29, p<.05]$. Moreover, the stimulus augmented same-consequent responding relative to the ITI rate $[T(16)=19, p<.01]$. The response whose anticipation was the same as the reinforcer associated with the stimulus was not significantly altered from its baseline rate.

Testing of the auditory stimuli with the lever and chain found additional evidence that a stimulus promotes the likelihood of a response with which it shares a reinforcer. Each auditory stimulus had one response reinforced in its presence. However, the design called for the response trained in one auditory stimulus to be followed by the outcome associated with the other auditory stimulus. To the degree that the $\mathrm{R}-\mathrm{O}$ association governs performance, one might expect the occurrence of the response to be well promoted by the stimulus present during the training of the other response. During this test the mean responses per minute were $1.3,2.1$, and 2.5 for the ITI, the stimulus with which the response was trained, and the stimulus with which the other response was trained. Response rates during the stimuli differed from that during the ITI $\left[T_{\mathrm{s}}(16)<8, p<.05\right]$, but they did not differ from each other. Of particular interest is the finding that the auditory stimulus in the presence of which one response was reinforced was capable of promoting the occurrence of the other response. Given earlier data (e.g., Colwill \& 


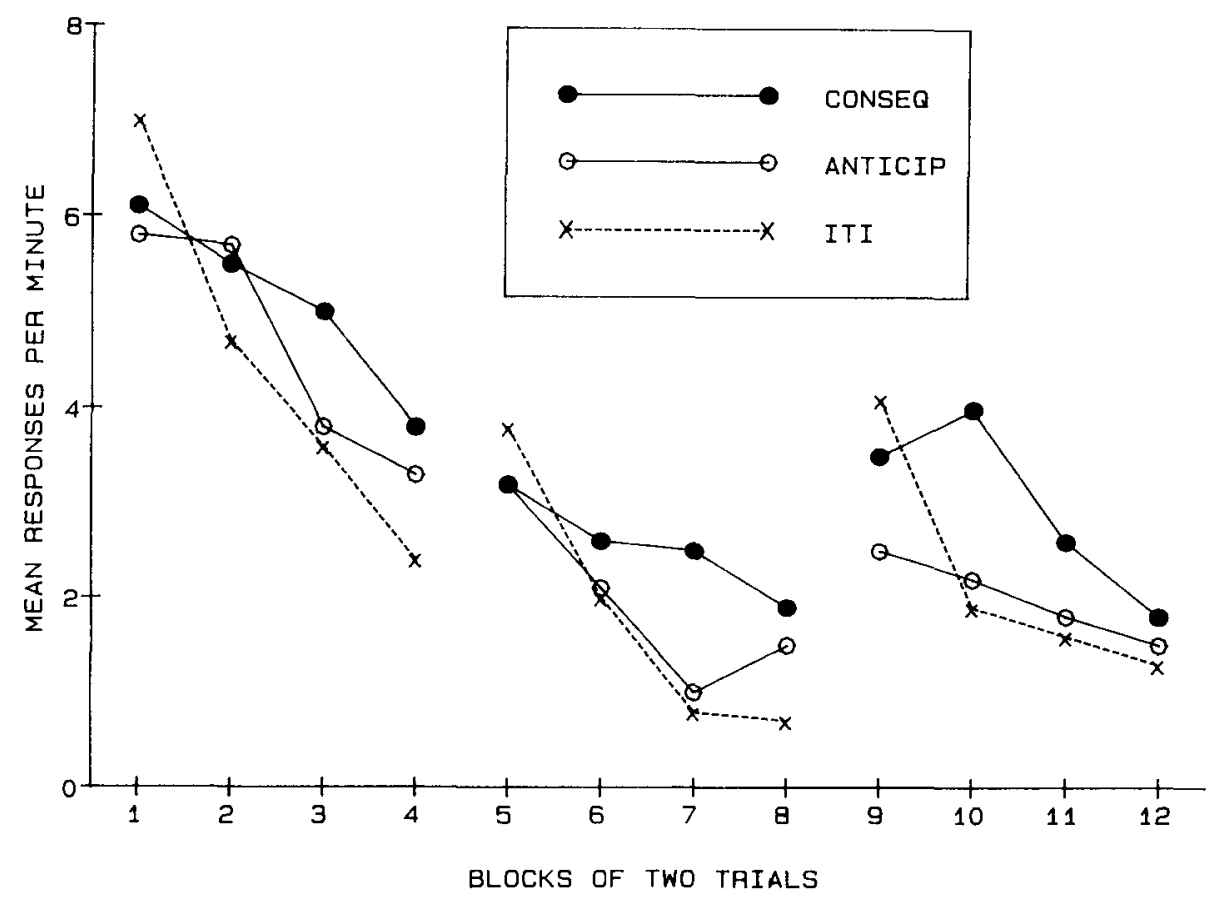

Figure 6. Test results of Experiment 4. Mean responses per minute are shown for the three test sessions with the two visual stimuli. Responding during a stimulus is shown separately when the CONSEQuent reinforcer was the same for both the response and the stimulus (filled circles), and when the ANTICIPated reinforcer was the same for both the response and the stimulus (open circles); responding during the ITI is also shown (dashed line).

Rescorla, 1988), that promotion seems best interpreted in terms of transfer based on the stimulus's sharing an outcome with the response.

\section{GENERAL DISCUSSION}

In these experiments, encoding of the reinforcer in the course of instrumental learning was investigated. They contrasted one condition in which a reinforcer was established as a consequence of an instrumental response with another condition in which the expectation of a reinforcer served as an antecedent stimulus to that instrumental response. Experiments 1 and 2 showed that changing the value of the reinforcer that followed a response had a profound depressive effect on performance of that response, whereas changing the value of the antecedent reinforcer had negligible impact. Experiment 3 strengthened the conclusion that devaluation of the antecedent reinforcer has little effect on instrumental performance. Experiment 4 showed that a discriminative stimulus associated with a reinforcer will act preferentially on a response that has the same reinforcer as a consequence rather than as an antecedent. These results encourage the view that reinforcers are encoded as consequences of the instrumental responses that produce them, and that those consequences then influence instrumental responding. At least with the small amounts of training used here, there is no compelling evidence for the alternative that $\mathrm{O}-\mathrm{R}$ associations are crucial determinants of instrumental behavior.
These results are not particularly congenial for standard two-process theories of instrumental behavior. Such theories agree in emphasizing the importance of $\mathrm{S}-\mathrm{O}$ associations in governing responding; but they do so in two quite different ways. On the one hand, some authors (e.g., Trapold \& Overmier, 1972) describe that association as playing a mediational role in which the anticipated $O$ serves as a stimulus controlling the instrumental response. On the other hand, others (e.g., Rescorla \& Solomon, 1967) have seen that $\mathrm{S}-\mathrm{O}$ association as having motivational properties that amplify instrumental behavior. Theories of the first type are embarrassed by the present finding that it is not the reinforcer anticipated prior to a response that governs the effects of devaluation and transfer. Theories of the second type find awkward the result, described here and elsewhere (e.g., Baxter \& Zamble, 1982; Colwill \& Rescorla, 1988; Kruse, Overmier, Konz, \& Rokke, 1983), that the impact of a stimulus upon a response depends on their sharing a common reinforcing outcome. Such theories have usually assumed that a stimulus associated with an outcome has a rather general motivational impact on responding that is relatively independent of the detailed identity of that outcome.

However, it may be noted that this assumption of generality is not an inherent feature of a motivational two-process theory. An account that combined a reinforcer-specific motivational state with an assumption of a responsereinforcer association could readily generate the results of the present experiments. Indeed, such a theory would 
be similar to the threshold-shifting account given by Colwill and Rescorla (1988). They have suggested that the role of the $\mathrm{S}-\mathrm{O}$ association is to allow $\mathrm{S}$ to lower the threshold for activation of the representation of the reinforcing outcome. That lowered threshold increases the ease with which a response associated with that outcome can succeed in activating its representation. Other evidence (e.g., Colwill \& Rescorla, 1985) suggests that performance of a response depends on its ability to activate representations of valued outcomes. Consequently, a stimulus that improves access to an outcome would effectively amplify the likelihood of instrumental responses associated with that outcome. In effect, such a stimulus would have a reinforcer-specific motivational effect on performance. Of course, within such an account, response-reinforcer associations play a primary role in governing instrumental performance.

These experiments reveal two additional features of response-reinforcer relations that are worth noting. First, they suggest that such associations develop with remarkable speed. Both the reinforcer devaluation and the stimulus-transfer techniques detected learning resulting from only a few (between 15 and 29) response-reinforcer conjunctions. Indeed, it seems probable that they would have detected learning with yet fewer occasions on which the response was followed by a reinforcer. Second, the development of a particular response-outcome association may not always be directly reflected in ongoing performance. In the present experiments, the substitution of one response-contingent outcome for another produced no marked performance effects. Yet subsequent devaluation and transfer tests revealed the development of response-outcome associations. This suggests that such detection procedures may provide superior means of assessing the nature of associations in instrumental behavior.

The present experiments were exclusively concerned with the case in which the expectation of a reinforcer is established as the antecedent stimulus for the instrumental response. However, it seems natural to ask whether a similar pattern of results might have been obtained had presentations of the reinforcer per se, rather than its expectancy, been established as the antecedent stimulus. Relevant to this issue is an experiment by Adams and Dickinson (1981), in which free deliveries of one reinforcer alternated with response-contingent deliveries of a different reinforcer. They found that poisoning of the contingent reinforcer was more effective in suppressing responding than poisoning of the reinforcer that regularly preceded a response. Thus, despite a number of demonstrations that reinforcers may serve as discriminative stimuli for responding (e.g., Capaldi, 1967; Rescorla \& Skucy, 1969), compared with the consequences of responding, they may be relatively less important in guiding instrumental performance.

This observation fits with the primary conclusion of the present paper that it is the association between the response and its consequent outcome, rather than that be- tween the anticipated outcome and the response, that primarily governs instrumental performance.

\section{REFERENCES}

Adams, C. D., \& Dickinson, A. (1981). Instrumental responding following reinforcer devaluation. Quarterly Joumal of Experimental Psychology, 33B, 109-121.

Asratyan, E. A. (1974). Conditioned reflex theory and motivational behavior. Acta Neurobiologiae Experimentalis, 34, 15-31.

BAXTER, D. J., \& Zamble, E. (1982). Reinforcer and response specificity in appetitive transfer of control. Animal Learning \& Behavior 10, 201-210.

Bolles, R. C. (1972). Reinforcement, expectancy, and learning. Psychological Review, 79, 394-409.

Brodigan, D. L., \& Peterson, G. B. (1976). Two-choice conditional discrimination performance of pigeons as a function of reward expectancy, prechoice delay and domesticity. Animal Learning \& Behavior, 4, 121-124.

CAPAI.DI, E. J. (1967). A sequential hypothesis of instrumental learning. In K. W. Spence \& J. T. Spence (Eds.), The psychology of learning and motivation (Vol. 1, pp. 67-156). New York: Academic Press

Colwill, R. M., \& Rescorla, R. A. (1985). Post-conditioning devaluation of a reinforcer affects instrumental responding. Journal of $E x$ perimental Psychology: Animal Behavior Processes, 11, 120-132.

Colwill, R. M., Rescorla, R. A. (1986). Associative structures in instrumental learning. In G. H. Bower (Ed.), The psychology of learning and motivation (Vol. 20, pp. 55-104). New York: Academic Press.

Colwill, R. M., \& Rescorla, R. A. (1988). Associations between the discriminative stimulus and the reinforcer in instrumental learning. Joumal of Experimental Psychology: Animal Behavior Processes, 14, 155-164.

KONORSK, J. (1948). Conditioned reflexes and neuron organization. Cambridge, England: Cambridge University Press.

Kruse, J. M., Overmier, J. B., Konz, W. A., \& Rokke, E. (1983). Pavlovian conditioned stimulus effects upon instrumental choice behavior are reinforcer specific. Learning \& Motivation, 14, 165-181.

Mackintosh, N. J., \& Dickinson, A. (1979). Instrumental (Type II) conditioning. In A. Dickinson \& R. A. Boakes (Eds.), Mechanisms of learning and motivation (pp. 143-167). Hillsdale, NJ: Erlbaum.

Overmier, J. B., Bull, J. A., III, * Trapold, M. A. (1971). Discriminative cue properties of different fears and their role in response selection in dogs. Journal of Comparative \& Physiological Psychology, 76, 478-482.

Peterson, G. B., Wheeler, R. L., \& Trapold, M. A. (1980). Enhancement of pigeons' conditional discrimination performance by expectancies of reinforcement and nonreinforcement. Animal Learning \& Behavior, 8, 22-30.

Rescorla, R. A., \& Skucy, J. C. (1969). Effect of responseindependent reinforcers during extinction. Journal of Comparative \& Physiological Psychology, 67, 381-389.

Rescorla, R. A., Solomon, R. L. (1967). Two-process learning theory: Relationships between Pavlovian conditioning and instrumental learning. Psychological Review, 74, 151-182.

SPENCE, K. W. (1956). Behavior theory and conditioning. New Haven, CT: Yale University Press.

Tolman, E. C. (1933). Sign-Gestalt or conditioned reflex? Psychological Review, 40, 246-255.

TRAPOLd, M. A. (1970). Are expectancies based upon different positive reinforcing events discriminably different? Learning \& Motivation, 1, 129-140.

Trapold, M. A., \& Overmier, J. B. (1972). The second learning process in instrumental learning. In A. A. Black \& W. F. Prokasy (Eds.), Classical Conditioning: II. Current research and theory (pp. 427-452). New York: Appleton-Century-Crofts.

(Manuscript received August 25, 1988; revision accepted for publication January $15,1989$. 